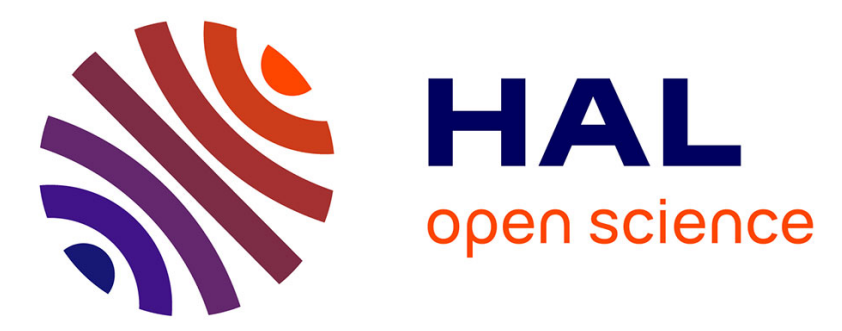

\title{
Uréides et asparagine chez deux variétés de soja cultivées au champ: stockage dans la tige et utilisation
}

Eric Serres, Jean Calmés, Guy Viala, Gérard Cavalié

\section{To cite this version:}

Eric Serres, Jean Calmés, Guy Viala, Gérard Cavalié. Uréides et asparagine chez deux variétés de soja cultivées au champ: stockage dans la tige et utilisation. Agronomie, 1985, 5 (10), pp.899-904. hal-00884719

\section{HAL Id: hal-00884719 https://hal.science/hal-00884719}

Submitted on 1 Jan 1985

HAL is a multi-disciplinary open access archive for the deposit and dissemination of scientific research documents, whether they are published or not. The documents may come from teaching and research institutions in France or abroad, or from public or private research centers.
L'archive ouverte pluridisciplinaire HAL, est destinée au dépôt et à la diffusion de documents scientifiques de niveau recherche, publiés ou non, émanant des établissements d'enseignement et de recherche français ou étrangers, des laboratoires publics ou privés. 


\title{
Uréides et asparagine chez deux variétés de soja cultivées au champ : stockage dans la tige et uti- lisation
}

\author{
Eric SERRES, Jean CALMÉS, Guy VIALA \& Gérard CAVALIÉ \\ Centre de Physiologie végétale, Laboratoire associé au C.N.R.S. $n^{\circ} 241$, Université Paul Sabatier, 118, route \\ de Narbonne, 31062 Toulouse Cedex
}

\begin{abstract}
Une étude des uréides glyoxyliques et de l'asparagine est réalisée sur 2 variétés de soja cultivées au champ, dont la croissance et la floraison sont de type indéterminé (« Amsoy 71 ») et de type déterminé ( $M 13$ »). Les substances azotées s'accumulent dans la tige : les entre-nœuds les plus riches se situent dans la région médiane pour " Amsoy 71 » ou apicale pour «M 13 ». Dans les 2 cas, ils correspondent à la zone qui produit le plus grand nombre de gousses. Les réserves caulinaires augmentent de la floraison jusqu'au début du grossissement des graines puis elles diminuent. Toutefois, la formation puis la disparition des réserves d'asparagine sont plus précoces que celles des uréides. Il existe également un décalage dans l'évolution journalière des teneurs de la sève. Ces différences sont probablement dues au fait que les 2 composés n'ont pas la même origine. L'abondance des uréides peut servir d'indicateur pour la fixation symbiotique de l'azote.
\end{abstract}

Mots clés additionnels : Acide allantö̈que, allantoine, nutrition azotée, Glycine max.

Glyoxylic ureides and asparagine were measured in field-grown soybean (Glycine max (L.) Merr.), cv. "Amsoy » (indeterminate growth type) and cv. « M $13 »$ (determinate growth type). Nitrogenous compounds were abundant in the stem : highest amounts were found in the middle part of the plant in « Amsoy 71 » and in the apical part in « M 13 » genotype. In each case, these areas bore the largest number of pods. The amounts in the stem increased from flowering stage until the beginning of seed filling, then decreased. However asparagine content rose and declined earlier than that of ureides. The maximal content of the two compounds in sap was shifted by two hours during the course of a day. These data suggest a different origin for these two nitrogenous compounds. Ureide content may used as an indicator of symbiotic nitrogen fixation.

Additional key words : Allantoic acid, allantoin, nitrogen nutrition, Glycine max.

\section{INTRODUCTION}

Le soja, Glycine $\max (\mathrm{L}$.$) Merr., comme toutes les$ légumineuses, dispose d'une double source d'azote, l'azote minéral du sol et l'azote atmosphérique fixé par Rhizobium. La nitrate réductase des racines et surtout la nitrogénase des nodosités sont à l'origine de l'azote organique qui circule ensuite dans la tige sous forme d'asparagine et d'uréides glyoxyliques.

La biogenèse de ces composés a été beaucoup étudiée. L'asparagine est synthétisée à partir de l'acide aspartique, grâce à l'asparagine synthétase présente dans de nombreux tissus, y compris les nodosités (FUJIHARA \& YAMAGUCHI, 1980 ; REYNOLDS et al., 1982). L'origine des uréides a été en partie élucidée par l'utilisation de l'azote 15 (OHYAMA \& KumA-
ZAWA, 1979). L'azote atmosphérique, réduit en $\mathrm{NH}_{4}$ par la nitrogénase, est intégré dans les bases puriques nouvellement synthétisées (BOLAND \& SCHUBERT, 1983) ; celles-ci sont progressivement transformées en allantoïne, puis en acide allantoïque.

L'intérêt pour les uréides et l'asparagine n'a pas cessé de grandir, comme en témoignent de nombreux articles de synthèse (LEA \& MIFLIN, 1980 ; THOMAS \& SCHRADER, 1981 ; Mc NEIL, 1982; JOLIVET \& Mossé, 1982) et des travaux récents (ZENGBÉ \& SALSAC, 1983 ; CALMÉS et al., 1983 ; RAINBIRD et al., 1984 ; ARGILlIER et al., 1984). Toutefois, la répartition de ces composés azotés entre les divers entrenœuds de la tige a été peu étudiée. Ce travail se propose de la préciser, depuis la floraison jusqu'à la maturité des graines, chez 2 variétés de soja. 


\section{MATÉRIEL ET MÉTHODES}

Les 2 variétés de soja étudiées sont : «Amsoy 71 » dont la croissance et la floraison sont de type indéterminé et « M 13 », de type déterminé, caractérisé par un nombre défini d'entre-nœuds et un développement simultané de toutes les fleurs. La durée du cycle végétatif de la $2^{\mathrm{e}}$ variété est plus longue d'environ $15 \mathrm{j}$.

Les cultures sont réalisées au champ sur une terre alluviale de la vallée de la Garonne, irriguée, bien pourvue en éléments minéraux $(K, P)$ mais n'ayant reçu aucun apport d'engrais azotés. Les semences sont préalablement enrobées de tourbe contenant Rhizobium japonicum (Inoculum Biodoz Soja stabilisé, Lipha 115, Lyon).

Pour chacune des 2 variétés, des lots de 3 plantes, bien représentatives du peuplement végétal, sont prélevés à 6 stades successifs de la fructification définis suivant les critères de FEHR et al. (1971). Les échantillons sont conservés au froid, à $-18^{\circ} \mathrm{C}$, avant d'être analysés. Pour les prélèvements de sève, les tiges de 3 pieds sont sectionnées au-dessus du bulbe cotylédonaire et un dispositif spécial comprenant un manchon de caoutchouc surmonté d'un tube de verre permet de récupérer les exsudats.

La teneur en asparagine est déterminée après hydrolyse enzymatique de l'amide en aspartate et ammoniac et mesure colorimétrique $(436 \mathrm{~nm})$ de ce dernier composé en présence de réactif de NESSLER.
Les uréides sont dosés par la méthode de FOSSE et al. (1931), améliorée par BRUNEL \& CAPELLE (1959). Elle est basée sur le dosage du glyoxylate provenant de l'hydrolyse chimique de l'acide allantoïque et de l'allantoïne (SERRES, 1982). Le glyoxylate est transformé en phénylhydrazone qui, oxydée en présence de ferricyanure, forme un complexe rouge de 1-5 diphénylformazan (MATSUi et al., 1965) ; l'intensité de la coloration est mesurée à $520 \mathrm{~nm}$ (MATSUMoto et al., 1977).

La leghémoglobine des nodosités est dosée par fluorimétrie au moyen d'un spectrofluorimètre Jobin \& Yvon JY 3 D. Les longueurs d'onde optimales sont $403 \mathrm{~nm}$ à l'excitation et $601 \mathrm{~nm}$ à l'émission.

\section{RÉSULTATS}

\section{A. Répartition dans la tige de la variété "Amsoy $71 »$}

De la floraison à la maturation des graines $\left(7^{\mathrm{e}}\right.$ à $13^{\mathrm{e}}$ semaines après le semis) l'asparagine et les uréides s'accumulent dans tous les entre-nœuds de la tige. La prépondérance de l'azote uréidique par rapport à l'azote de l'asparagine augmente au cours de cette période (fig. 1). Les entre-nœuds inférieurs qui portent les ramifications s'appauvrissent dès la $8^{\mathrm{e}}$ semaine

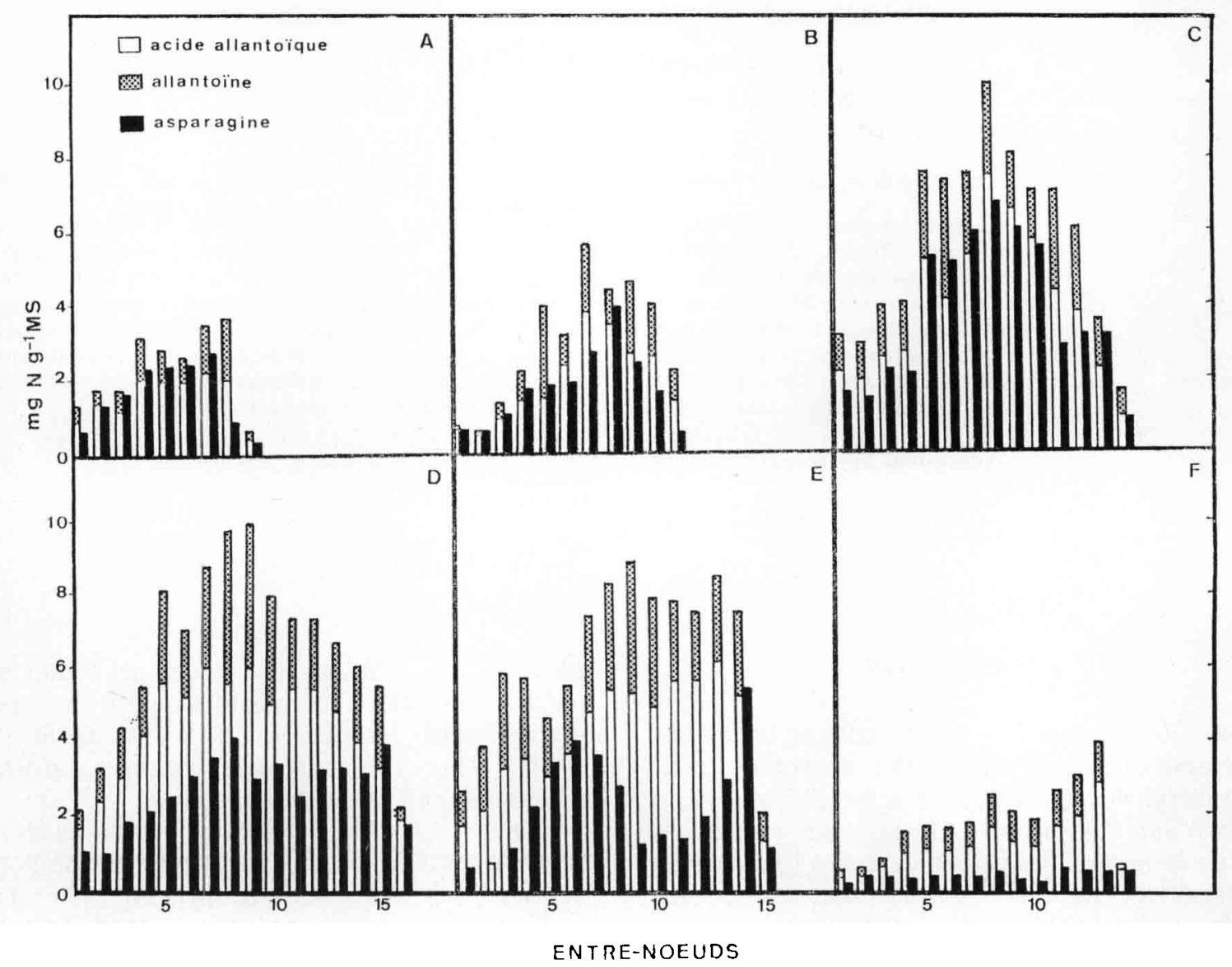

Figure 1

Teneurs en uréides et asparagine ( $m g$ N.g $M S^{-1}$ ) des entre-nouds de la tige comptés à partir du bas. Variété «Amsoy 71 "(moyenne de 3 plantes). A, floraison ( $\gamma^{e}$ semaine après le semis) ; $B$, formation des gousses ( $8^{e}$ semaine); $C$, début du grossissement des graines (10e semaine) : $D$, grossissement des graines ( $12^{e}$ semaine) $E$, maturation des graines (13e semaine); $F$, maturité $\left(15^{e}\right.$ semaine).
Changes in the stem contents of ureides and asparagine (mg N.g $\left.D W^{-1}\right)$. Internodes numbered from the base. Genotype "Amsoy $7 I$ " (mean of three plants). A, flowering (7 weeks a.s.); $B$, pod setling ( 8 weeks) ; $C$, beginning of seed filling ( 10 weeks) $D$, seed filling (12 weeks); $E$, seed maturation (13 weeks); $F$, maturity (15 weeks). 
alors que s'enrichissent ceux du milieu qui portent le plus grand nombre de fructifications. Le maximum d'azote organique est accumulé au début du grossissement des graines où la teneur en asparagine est maximale $(6,8 \mathrm{mg}$ d'azote par $\mathrm{g}$ de matière fraîche dans les entre-nœuds médians). A ce stade le rapport $\mathrm{N}$ uréidique/ $\mathrm{N}$ de l'asparagine est d'environ 1,4; ensuite les teneurs en asparagine diminuent tandis que celles des uréides deviennent maximales $\left(10 \mathrm{mg} \mathrm{N} \cdot \mathrm{g}^{-1}\right.$ de matière fraîche) et le rapport des 2 formes d'azote atteint 2,6.

A la fin de la maturité des graines (15 $5^{\text {e }}$ semaine) les réserves en asparagine ont disparu en quasi-totalité, celles en uréides ont fortement diminué.

La figure 2 montre que les teneurs en uréides de la tige et la teneur en leghémoglobine des nodosités évoluent de manière très comparable.

\section{B. Répartition dans la tige de la variété «M 13 »}

Les teneurs en asparagine sont voisines de celles de la variété "Amsoy 71 ». En revanche, la richesse en uréides est plus élevée (fig. 3). Le maximum d'uréides et d'asparagine intervient au début du grossissement des graines $\left(12^{\mathrm{e}}\right.$ semaine après le semis (fig. $3 \mathrm{C}$ ). Contrairement à la variété «Amsoy 71 », les entrenœuds les plus riches sont situés vers l'extrémité supérieure où les gousses sont les plus nombreuses et les mieux remplies en graines. Il s'est établi un gradient croissant dans les teneurs depuis la base jusqu'à l'apex.

$\mathrm{Au}$ cours des semaines suivantes, comme pour la variété « Amsoy 71 », on observe (fig. 3 D) une utilisation massive, de l'asparagine d'abord, des uréides ensuite. Toutefois, la mobilisation de ces derniers est moins complète, surtout pour les entre-nœuds apicaux (fig. 3 F).

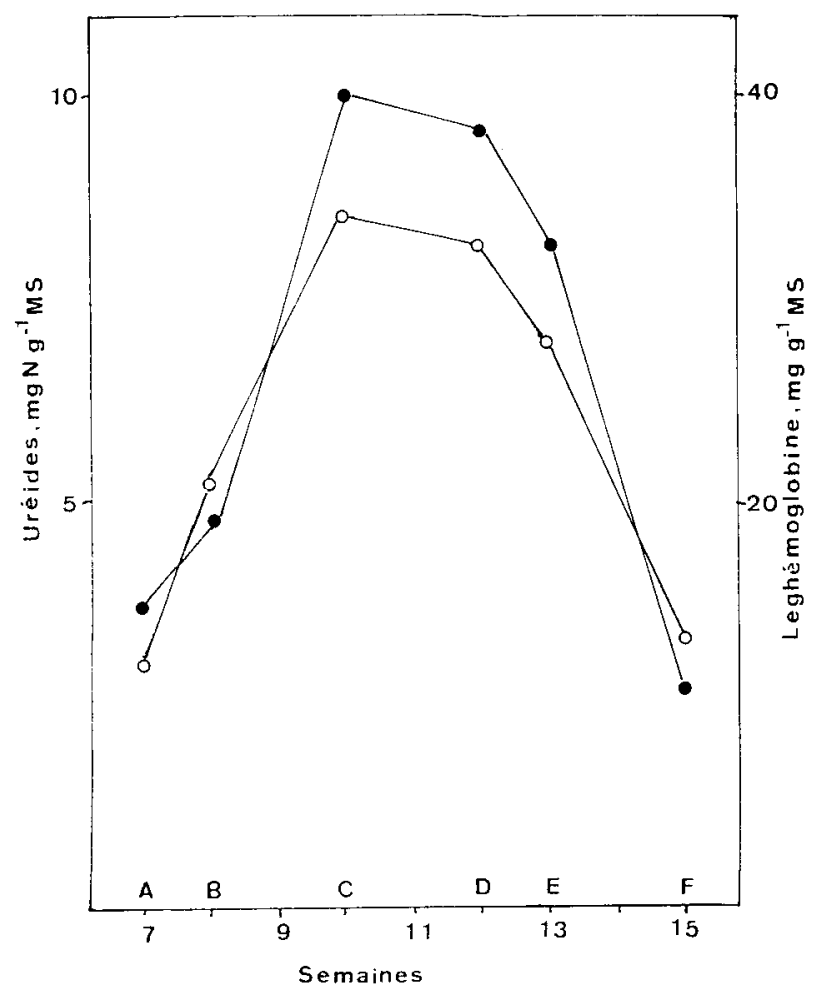

Figure 2

Evolution des teneurs en uréides $\bullet$ ( $8^{e}$ entre-nœud de la tige) et en leghémoglobine des nodosités pour la variété "Amsoy 7I". Mêmes stades que pour la figure 1 .

Change in ureides $\bullet$ (8th internode of the stem) and leghaemoglobin $\bigcirc$ (nodules) contents. "Amsoy 71 ". Same stages as 1.

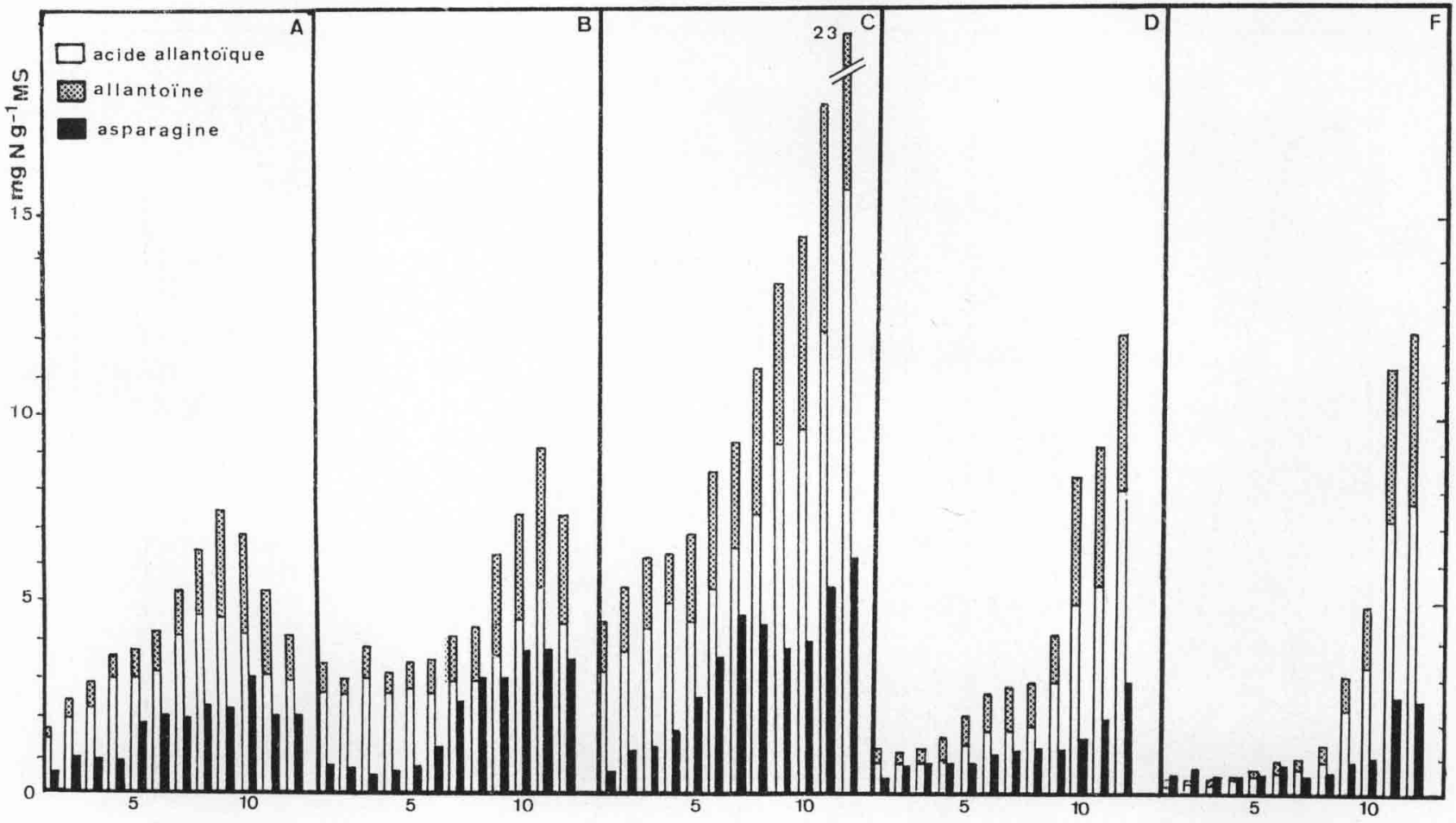

Figure 3

Teneurs en uréides et asparagine ( $m g N . g M^{-1}$ ) des entre-nœuds de la tige. Variété "M 13 " (moyenne de 3 plantes). A, floraison (9e semaine après le semis); $B$, formation des gousses $\left(10^{e}\right.$ semaine) ; $C$, début du grossissement des graines $\left(12^{e}\right.$ semaine $; D$, maturation des graines (14 semaine); $F$, maturité (16e semaine).
ENTRE-NOEUDS

Changes in the stem contents of ureides and asparagine (mg N.g $D W^{-1}$ ). Genotype $M 13$ (mean of three plants). A, flowering (9 weeks a.s.); $B$, pod setting (10 weeks) ; $C$, beginning of seed filling (12 weeks); D, seed maturation (14 weeks); F, maturity (16 weeks). 
Une décortication annulaire permet de séparer le parenchyme ligneux de l'écorce : du point de vue anatomique, cette dernière correspond au parenchyme cortical et à la zone libérienne. Une étude analytique, effectuée à l'époque des plus fortes teneurs en uréides et asparagine, montre que la majeure partie des composés azotés se localise dans le parenchyme ligneux : 75 p. 100 dans le cas des uréides et 84 p. 100 pour l'asparagine (tabl. 1).

\section{Composition de la sève en uréides et asparagine}

L'évolution de l'azote organique contenu dans la sève prélevée au niveau du bulbe cotylédonaire est suivie avec la variété «M 13 ». Les teneurs en uréides varient au cours de la journée (fig. 4 A) : très élevées $\left(1,3 \mathrm{mg} \mathrm{N} \cdot \mathrm{ml}^{-1}\right)$ le matin vers $6 \mathrm{~h}$ (temps universel), elles deviennent 10 fois plus faibles entre 12 et $14 \mathrm{~h}$ lorsque le flux de sève est maximum $\left(6 \mathrm{ml}\right.$.plante $\left.{ }^{-1} \cdot \mathrm{h}^{-1}\right)$. Le calcul des quantités d'azote uréidique transportées (fig. $4 \mathrm{~B}$ ) montre qu'elles sont particulièrement importantes à $6 \mathrm{~h}\left(1,15 \mathrm{mg} \mathrm{N} . \mathrm{h}^{-1}\right)$ mais aussi à $12 \mathrm{~h}\left(0,9 \mathrm{mg} \mathrm{N} \cdot \mathrm{h}^{-1}\right)$; bien que très diluée, la sève transporte alors beaucoup d'uréides.

La teneur en asparagine est en moyenne 9 fois plus faible que celle des uréides. L'étude des variations jouı nalières montre que la teneur maximale se situe à $8 \mathrm{~h}$ (fig. $4 \mathrm{~A}$ ) ; il existe un décalage de $2 \mathrm{~h}$ par rapport aux uréides. Cependant la quantité transportée est surtout importante à $12 \mathrm{~h}$ (fig. $4 \mathrm{~B}$ ).

\section{TABLEAU 1}

Répartition des uréides et de l'asparagine entre le bois et l'écorce pour les entre-noeuds des étages 7 à 10 de la variété « $M 13$ », à l'époque du grossissement des graines (moyenne de 12 échantillons).

Distribution of ureides and asparagine between wood and bark for 7-10th internodes of genotype "M 13", at the seed-filling stage (mean of 12 samples).

\begin{tabular}{|c|c|c|c|c|}
\hline & \multicolumn{2}{|c|}{ Uréides } & \multicolumn{2}{|c|}{ Asparagine } \\
\hline & $\begin{array}{l}\text { Teneurs } \\
\text { mg.g MS }{ }^{-1}\end{array}$ & $\begin{array}{c}\text { Quantités } \\
\text { mg.organe }\end{array}$ & $\begin{array}{l}\text { Teneurs } \\
\text { mg.g MS }{ }^{-1}\end{array}$ & $\begin{array}{c}\text { Quantités } \\
\text { mg. organe }\end{array}$ \\
\hline Bois & $13,6 \pm 2,5$ & $15,5 \pm 2,9$ & $5,4 \pm 0,5$ & $6,1 \pm 0,6$ \\
\hline Ecorce & $9,6 \pm 1,8$ & $5,2 \pm 1,0$ & $2,2 \pm 0,3$ & $1,2 \pm 0,2$ \\
\hline
\end{tabular}

Cette heure de la journée apparaît propice pour une étude des variations au cours de la fructification (fig. $4 \mathrm{C}$ ). Les quantités d'uréides de la sève augmentent rapidement de la $7^{\mathrm{e}}$ à la $11^{\mathrm{e}}$ semaine après le semis. Elles présentent alors la valeur la plus élevée $(0,54 \mathrm{mg}$ $\mathrm{N}$. plante $\left.\mathrm{e}^{-1} \cdot \mathrm{h}^{-1}\right)$. Ensuite, elles diminuent régulièrement.

C'est au début de la floraison que la sève est la plus riche en asparagine : $0,21 \mathrm{mg} \mathrm{N}$.plante ${ }^{-1} \cdot \mathrm{h}^{-1}$; le rapport $\mathrm{N}$ uréidique/ $\mathrm{N}$ de l'asparagine est alors inférieur à l'unité. Mais la valeur du rapport augmente ensuite et dépasse 10 au début du grossissement des graines pour revenir à 3 à l'approche de la maturité.
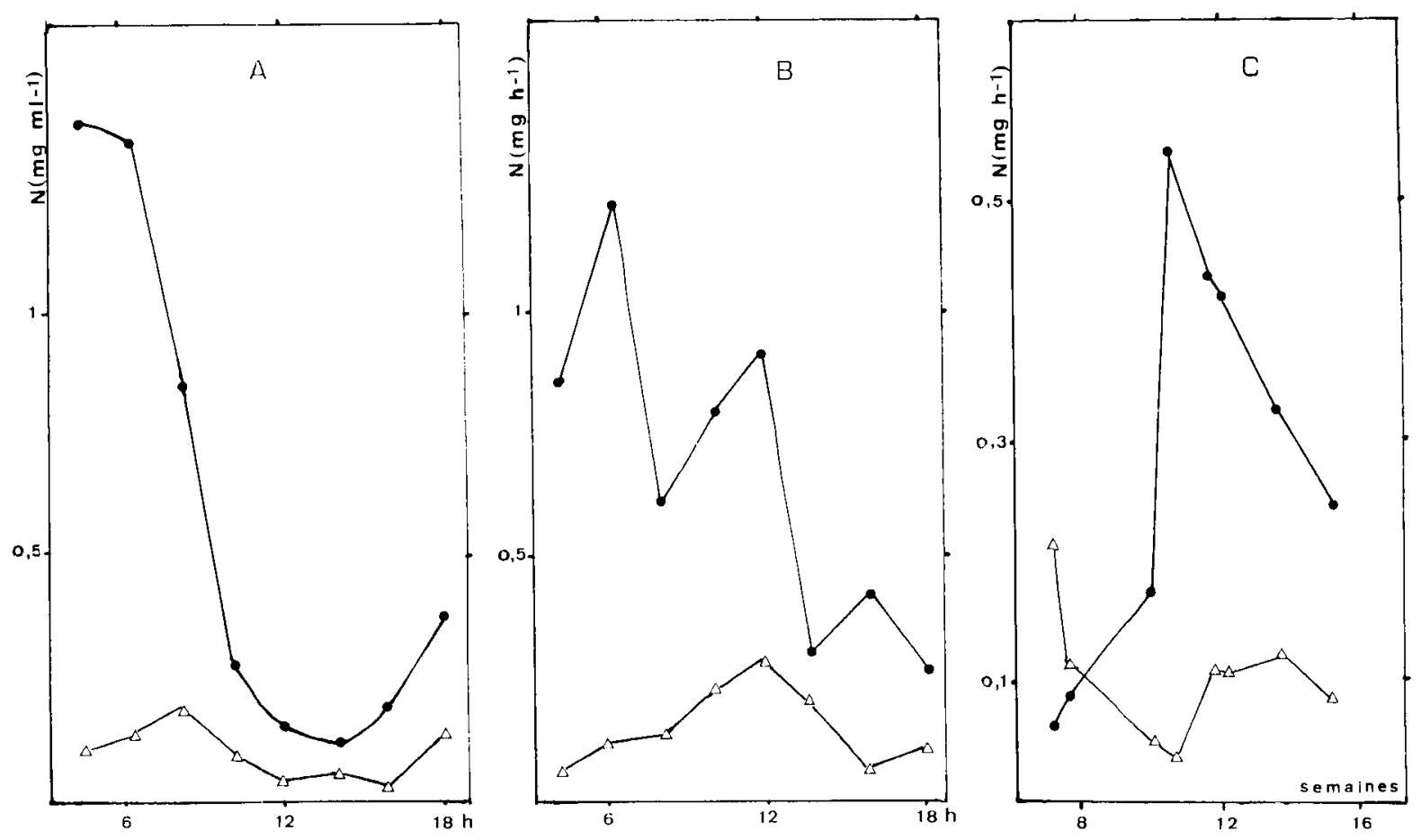

Figure 4

Uréides et asparagine dans la sève. Variété " M13" (moyenne de 3 plantes). $A$, variation journaliere des teneurs ( $m g \mathrm{~N} . \mathrm{ml}^{-1}$ ) pour les uréides $\bullet$ et l'asparagine $\triangle ; B$, variation journalière des quantités transportées ( $\left.m g \mathrm{~N} . h^{-1}\right) ; C$, variation des quantités transportées (mg $N . h^{-1}$ ) au cours de la période de fructification.

Ureides and asparagine in stem exudate. Genotype "M 13 "' (mean of 3 plants). A, daily rate ( $m g ~ N . \mathrm{ml}^{-1}$ ) for ureides - and asparagine $\triangle ; B$, daily translocated amounts (mg N. $\left.h^{-1}\right) ; C$, translocated amounts (mg N. $h^{-1}$ ) during reproductive development. 
TABLEAU 2

Distribution des uréides et de l'asparagine (mg N.g MS-1) dans les étages de rang 5 à 11 de la variété « Amsoy 71 » au début du grossissement des graines (moyenne de 18 échantillons).

Distribution of ureides and asparagine ( $m g \mathrm{~N} . \mathrm{g} \mathrm{DW} W^{-1}$ ) in the 5-11th foliar levels of genotype "Amsoy 71 ", at the beginning of the seed filling stage (mean of 18 samples).

\begin{tabular}{lcccccc}
\hline \hline & Entre-nœuds & Pétiole & Folioles & Cosses & Graines \\
\cline { 2 - 7 } & & & & & & \\
Uréides & $5,8 \pm 1,2$ & $0,91 \pm 0,20$ & $0,15 \pm 0,04$ & $7,54 \pm 0,62$ & $0,81 \pm 0,15$ \\
$\quad$ A. allantö̈que & $2,2 \pm 0,7$ & $0,70 \pm 0,15$ & $0,05 \pm 0,02$ & $1,31 \pm 0,20$ & $0,22 \pm 0,06$ \\
$\quad$ Allantö̈ne & $5,8 \pm 0,6$ & $0,61 \pm 0,12$ & $0,12 \pm 0,03$ & $4,66 \pm 0,42$ & $7,13 \pm 0,58$ \\
Asparagine & $0,97 \pm 0,25$ & $0,33 \pm 0,07$ & $0,89 \pm 0,19$ & $0,71 \pm 0,14$ & $0,75 \pm 0,16$ \\
Masse de matière sèche (en g) & & &
\end{tabular}

\section{Répartition des uréides et de l'asparagine dans la plante}

Les uréides et l'asparagine contenus dans les diverses parties de la plante sont analysés à partir de 3 pieds appartenant à la variété «Amsoy 71 », et ceci aux principaux stades de développement. Dans le système racinaire (racines et nodosités), les teneurs restent faibles; elles ne dépassent pas les valeurs suivantes : $0,6 \mathrm{mg}$ d'azote par $\mathrm{g}$ de matière sèche pour l'asparagine et 0,15 pour les uréides.

Dans les parties aériennes, le tableau 2 regroupe les teneurs concernant les 6 étages médians (rangs 5 à 11), à l'époque où la tige présente les teneurs maximales $\left(10^{\mathrm{e}}\right.$ semaine après le semis). Pour les uréides, la tige est l'organe le plus riche suivi de très près par les cosses ; viennent ensuite les pétioles et les graines avec des teneurs de 6 à 8 fois plus faibles, et, en dernier lieu, les folioles avec $0,2 \mathrm{mg}$ de $\mathrm{N}$ uréidique par $\mathrm{g}$ de matière fraîche, soit 50 fois moins que dans la tige. Dans tous les organes l'acide allantoïque est plus abondant que l'allantoïne.

Pour l'asparagine, la répartition est sensiblement la même que celle des uréides en ce qui concerne la tige, les cosses, les pétioles et les folioles ; en revanche, les graines sont particulièrement riches en cette amide.

Un bilan effectué à partir des données du tableau 2 montre que la tige contient la moitié environ des uréides et les $2 / 5$ de l'asparagine présents dans l'ensemble des étages médians. Ces deux substances constituent, dans la tige, près de 95 p. 100 de l'azote organique soluble ; des analyses complémentaires indiquent que le reste $(5 \mathrm{p} .100)$ correspond à des acides aminés libres : aspartate, glutamate, glutamine.

\section{DISCUSSION}

Les quantités d'asparagine et d'uréides, présentes dans la tige, évoluent de façon assez indépendante. Alnsi les analyses de sève font apparaître, d'une part, un décalage journalier de $2 \mathrm{~h}$ entre les teneurs maximales de ces composés et, d'autre part, une variation de leur importance respective au cours de la fructification, comme en témoignent les valeurs du rapport $\mathrm{N}$ uréidique/ $\mathrm{N}$ de l'asparagine. L'accumulation de l'asparagine dans la tige est plus précoce que celle des uréides et les. réserves en amide diminuent plus rapide- ment au cours de la période du grossissement des graines. Ces évolutions différentes sont à rapprocher d'autres faits expérimentaux. Les jeunes pieds non inoculés ou aux nodosités non encore fonctionnelles contiennent beaucoup d'asparagine et très peu d'uréides (SERRES, 1983). La sève de sojas nodulés, cultivés sans azote puis transférés sur milieu nitrique afin d'inhiber l'activité fixatrice, s'enrichit en asparagine (TRINCHANT \& RIGAUD, 1981, 1982 ; LAYZELL \& LA RUE, 1982).

L'ensemble de ces observations traduit une origine différente pour les 2 composés azotés. Il existe une corrélation directe entre l'accumulation caulinaire des uréides et les teneurs en leghémoglobine (SERRES, 1983). Bien que les sojas non inoculés renferment de faibles quantités d'uréides, l'abondance de ces composés paraît liée, avant tout, au bon fonctionnement des nodosités. En revanche, malgré la présence d'asparagine synthétase dans les nodosités (STREETER, 1974 ; FuJihara \& YAMAguchi, 1980 ; Huber \& STREeTER, 1984), la majeure partie de l'azote amidé pourrait provenir, chez le soja, de la réduction du nitrate. La nitrate réductase, très active dans la feuille (OBATON et al., 1982), est également présente dans les racines (HARPER \& HAGEMAN, 1972).

De la floraison jusqu'au début du grossissement des graines, la tige accumule de plus en plus d'asparagine et d'uréides. Durant cette période, les quantités d'azote organique transportées par la sève sont supérieures aux besoins de la protéogenèse foliaire es des jeunes gousses. La tige devient un organe de stockage; les réserves sont particulièrement importantes dans les régions qui produisent le plus grand nombre de fructifications : région médiane pour la variété «Amsoy 71», partie apicale pour la variété « M $13 »$.

L'accumulation caulinaire de l'asparagine et des uréides, résultat d'un excédent des synthèses sur l'utilisation, peut être utilisée pour tester la bonne alimentation azotée de la plante. Ce test présente d'ailleurs une grande sensibilité puisque, au moment du développement des gousses, un ralentissement momentané dans le fonctionnement des nodosités entraîne aussitôt une diminution des teneurs en uréides dans la tige (SERRES, 1983). Donc cette teneur peut servir d'indicateur de la fixation symbiotique de l'azote (Mc ClURE et al., 1980; SHEARER et al., 1982) à condition toutefois d'analyser les entre-nœuds situés dans la zone de production maximale. Enfin, la valeur du 
rapport $\mathrm{N}$ uréidique/ $\mathrm{N}$ de l'asparagine renseigne sur l'importance de l'azote fixé par rapport à l'alimentation nitrique.

Reçu le 13 mai 1985. Accepté le 8 juillet 1985

\section{REMERCIEMENTS}

Nous sommes reconnaissants à Mlle A. Bouniols (I.N.R.A., Toulouse) et à M. le Professeur L. SALSAC (E.N.S.A., Montpellier) pour leurs discussions fructueuses et nous adressons nos remerciements à Gisèle BORDERIES pour sa collaboration technique efficace.

\section{RÉFÉRENCES BIBLIOGRAPHIQUES}

Argillier C., Zengbé M., Salsac L., 1984. Métabolisme des uréides chez le soja (Glycine max (L.) Merr.) et le pois (Pisum sativum L.). C. R. Acad. Sci., Paris, série III, 299, 63-68.

Boland M. J., Schubert K. R., 1983. Biosynthesis of purines by proplastid fraction soybean nodules. Arch. Biochem. Biophys., 220, 179-187.

Brunel A., Capelle G., 1947. Importance of glyoxylic acid in living organisms. I. Allantoin and allantoic acid in plants. Bull. Soc. Chim. Biol., 29, 427-444.

Calmés J., Serres E., Viala G., 1983. Métabolisme de l'asparagine dans les feuilles et les gousses de soja (Glycine max (L.) Merr.). C. R. Acad. Sci., Paris, série III, 296, 939-942.

Fehr W. R., Caviness C. E., Burmood D. J., Pennington J. S., 1971. Stages of development description for soybean, Glycine max. Crop Sci., 11, 929-931.

Fosse R., Brunel A., Thomas P. E., 1931. Analyse quantitative de très petites quantités d'allantö̈ne à de très grandes dilutions. Application à l'urine humaine. C. R. Acad. Sci., Paris, 192, 1615-1617.

Fujihara S., Yamaguchi M., 1980. Asparagine formation in soybean nodules. Plant Physiol, 66, 139-141.

Harper J. E., Hageman R. H., 1972. Canopy and seasonal profiles of nitrate reductase in soybean (Glycine max (L.) Merr.). Plant Physiol., 49, 146-154.

Huber T. A., Streeter J. G., 1984. Asparagine biosynthesis in soybean nodules. Plant Physiol, 74, 605-610.

Jolivet E., Mossé J., 1982. Non-protein nitrogenous compounds with particular attention to ureides. In Arora S. K. : "Chemistry and Biochemistry of Legumes », 195-216, Oxford IDH Publishing, New York, Bombay, Calcutta.

Layzell D. B., La Rue T. A., 1982. Modeling C and N transport to developing soybean fruits. Plant Physiol., 70, 1290-1298.

Lea P. I., Miflin B. J., 1980. Transport and metabolism of asparagine and other nitrogen compounds within the plant. In Miflin B. J. "The Biochemistry of Plants, Vol. 5. Amino acids and derivalives », 569-607, Academic Press, New York, London, 670 p.

Mac Clure P. R., Israel D. W., Volk P. J., 1980. Evaluation of the relative ureide content of xylem sap as an indicator of nitrogen fixation in soybean. Greenhouse studies. Plant Physiol., 66, 720-725.

Mac Neil D. L., 1982. Quantification of symbiotic nitrogen fixation using ureides: a review. Biol. Nitrogen fixation Technol. Trop. Agric., 609-617.

Matsui M., Okada M., Ishidate M., 1965. Structure of the principal coloured product formed in the determination of glyoxylic acid. Anal. Biochem., 12, 143-149.

Matsumoto T., Yatazawa M., Yamamoto Y., 1977. Distribution and change in the contents of allantoin and allantoic acid in developing nodulating and non nodulation soybean plants. Plant Cell Physiol., 18, 353-359.

Obaton M., Miquel M., Robin P., Conejero G., Domenach A. M., Bardin R., 1982. Influence du déficit hydrique sur l'activité nitrate réductase et nitrogenase chez le soja (Glycine $\max$ (L.) Merr., var. «Hodgson »). C. R. Acad. Sci., Paris, série III, 294, 1007-1012.

Ohyama T., Kumazawa K., 1979. Assimilation and transport of nitrogenous compounds originated from ${ }^{15} \mathrm{~N}$ fixation and ${ }^{14} \mathrm{NO}_{3}$ assimilation. Soil Sci. Plant Nutr., 25, 9-19.

Rainbird R. M., Thorne J. H., Hardy R. W. F., 1984. Role of amides, amino acids and ureides in the nutrition of developing soybean seeds. Plant Physiol., 74, 329-334.

Reynolds P. H. S., Blevins D. G., Boland M. J., Schubert K. R., Randall D. D., 1982. Enzymes of ammonia assimilation in legumes nodules: a comparison between ureide and amide transporting plants. Physiol. Plant, 55, 255-260.

Serres E., 1982. Variations des uréides glyoxyliques au cours d'un cycle de développement chez le soja (Glycine $\max (\mathrm{L}$.) Merr.). C. $R$. Acad. Sci., Paris, série III, 295, 143-146.

Serres E., 1983. Uréides et asparagine du soja, leur rôle dans le transport de l'azote. Thèse Doct. $3^{\mathrm{e}}$ cycle, U.P.S., Toulouse, $67 \mathrm{p}$.

Shearer G., Feldman L., Bryan B. A., Skeeters J. L., Kohl D. H., Amarger N., Mariotti F., Mariotti A., $1982 .{ }^{15} \mathrm{~N}$ abundance of nodules as an indicator of $\mathrm{N}$ metabolism in $\mathrm{N}_{2}$-fixing plants. Plant Physiol., 70, 465-468.

Streeter J. G., 1974. Asparaginase and asparagine transaminase in soybean leaves and root nodules. Plant Physiol., 60, 235-239.

Thomas R. J., Schrader L. E., 1981. Ureide metabolism in higher plants, a review. Phytochem., 20, 361-371.

Trinchant J. C., Rigaud J., 1981. Acetylene reduction and respiration of bacteroids isolated from French beans receiving nitrate. Physiol. Plant., 53, 511-517.

Trinchant J. C., Rigaud J., 1982. Nitrite and nitric oxide as inhibitors of nitrogenase from soybean bacteroids. App. Environmental Microbiol., 44, 1385-1388.

Zengbé M., Salsac L., 1983. Variations des teneurs en uréides et enzymes du catabolisme purique chez Glycine max. Physiol. Vég., 21, 67-76. 